\title{
Criterion shifts in the measurement of tactile masking ${ }^{1}$
}

\author{
GEORGE A. GESCHEIDER, ${ }^{2}$ DANIEL D. HERMAN, AND JEFFREY N. PHILLIPS
}

HAMILTON COLLEGE

A series of experiments was conducted to determine the effects of method of threshold measurement on the amount of threshold shift produced by a masking stimulus. The amount of contralateral tactile masking of the fingertip was found to be much greater when thresholds were measured by the Békésy tracking method than when measured by a forced-choice method. When the Békésy tracking procedure was modified so that each observation interval containing a brief stimulus was followed almost immediately by a second observation interval not containing a stimulus, the amount of masking was substantially reduced for both contralateral tactile masking and auditorytactile masking and corresponded almost exactly with the amount of masking measured by the forced-choice method. No such reduction in the amount of masking was obtained by using this procedure to measure ipsilateral tactile masking or auditory masking.

When a Békésy threshold-tracking method was employed, tactile thresholds were raised as much as $5 \mathrm{~dB}$ by presentation of an auditory click (Gescheider \& Niblette, 1967). This finding suggested that intense auditory stimulation can reduce the detectability of tactile stimuli. However, threshold measurements obtained by classical psychophysical procedures, including the Békésy tracking method, could be greatly affected by shifts in S's decision criterion as well as by changes in stimulus detectability. If the auditory masker produced an upward shift in S's criterion, a more intense signal would be required to exceed criterion and a higher threshold would be measured than when no masker was presented. Working within the framework of detection theory in subsequent experiments, we found that auditory stimulation both reduces the detectability of a tactile stimulus and induces $S$ to raise his judgment criterion (Gescheider, Barton, Bruce, Goldberg, \& Greenspan, 1969).

Auditory, visual, and tactile intramodality masking measured by classical psychophysical methods, generally assumed to be determined solely by reduced stimulus detectability, may also be a function of criterion shifts. In the present study the detection-theory analysis of masking was extended to include the measurement of masking within a modality. The purpose of the study was to compare the amounts of tactile, auditory, and auditory-tactile masking obtained by a classical psychophysical method with the amounts obtained by techniques that are insensitive to changes in S's judgment criterion.

\section{EXPERIMENT 1}

Sherrick (1964), employing the Békésy tracking technique to measure vibrotactile thresholds, found that an intense masking stimulus applied to the contralateral fingertip raised the threshold at the test site substantially. The results of other studies, however, indicate little or no contralateral tactile interaction (Gescheider \& Wright, 1968; Uttal \& Cook, 1964; Uttal \& Smith, 1967). Recently, on the other hand, Gilson (1969) found interaction as masking, and Craig (1968) found it as summation. The large amounts of contralateral tactile masking obtained by Sherrick may have resulted from the combined effects of decreased stimulus detectability and upward criterion shifting. A two-interval forced-choice procedure was therefore used to measure stimulus detectability under various conditions of contralateral tactile masking. The value of $\mathrm{d}^{\prime}$, an index of stimulus detectability, is independent of S's criterion and can be computed from the percentage of correct judgments obtained by the forced-choice procedure. The amount of contralateral tactile masking caused by reduced stimulus detectability was specified from the changes in $d^{\prime}$ observed under the various masking conditions.

\section{Method}

Subjects. Three male Hamilton College students with no previous experience in psychophysical experiments were employed as Ss. Prior to the experiment several sessions were devoted to training the $S s$ in the detection of vibrotactile stimuli applied to the index fingertips.

Apparatus. Sinusoidal electrical signals lasting $350 \mathrm{msec}$ generated by an oscillator (General Radio 1304-B) were applied to an electronic switch (Grason-Stadler 829E) to give a $150-\mathrm{Hz}$ signal with a rise and decay time of $25 \mathrm{msec}$. The output of the electronic switch was applied to a phase shifter (Grason-Stadler E3520B) adjusted to provide two independent signals in phase when they were applied to two vibrators (Goodmans V-47). In each of the two channels the signal passed through a decade attenuator box and an impedance matching transformer before being applied to the vibrator.

In a small audiometric testing room (Industrial Acoustics Company), S made judgments of the presence or absence of the vibrotactile signal on his right index fingertip. With his hands resting on his knees, $S$ extended his left and right index fingertips to make contact with the vibrators. To insure constant mechanical coupling between vibrators and fingertips, pressure of the $1 / 4$-in.-diam contactor on the fingertips was maintained relatively constant at about $30 \mathrm{~g}$ by counterweighting the shafts on which the vibrators were attached. S was informed of an observation interval by means of a voltmeter, the pointer of which was deflected for $350 \mathrm{msec}$. Each trial consisted of two such observation intervals separated by $2 \mathrm{sec}$, and $S$ attempted to report which of the intervals contained a vibration on his right index fingertip. One observation interval contained the masker on the left index fingertip and the test stimulus on the right index fingertip and the other observation interval contained only the masker on the left index fingertip. On control trials the test stimulus alone was presented in one of the observation intervals.

The experimental design was a 4 by 5 factorial manipulation of test-stimulus intensity and masker intensity. The intensity levels of the test stimulus and masking stimulus were relative to each S's absolute threshold measured by the method of limits prior to the experiment. The intensity levels of the test stimuli were $-3,0,3$, and $6 \mathrm{~dB} S \mathrm{SL}$ and the intensity levels of the masking stimuli were 10,20 , 30 , and $40 \mathrm{~dB} \mathrm{SL}$

Within a block of 20 trials $S$ made judgments of test stimuli of one of the four intensity levels under one of the frve masking conditions (maskers of 10, 20, 30, $40 \mathrm{~dB}$ SL and no masker). Within a session 10 blocks of 20 trials were given, one for each of the five masking conditions at each of two test-stimulus intensities. In the following session 20 trials for each of the five masking conditions for the other two test-stimulus intensities were given. The order in which the test stimuli were administered over the two sessions was random, as was the order in which the masking conditions were given. A total of 


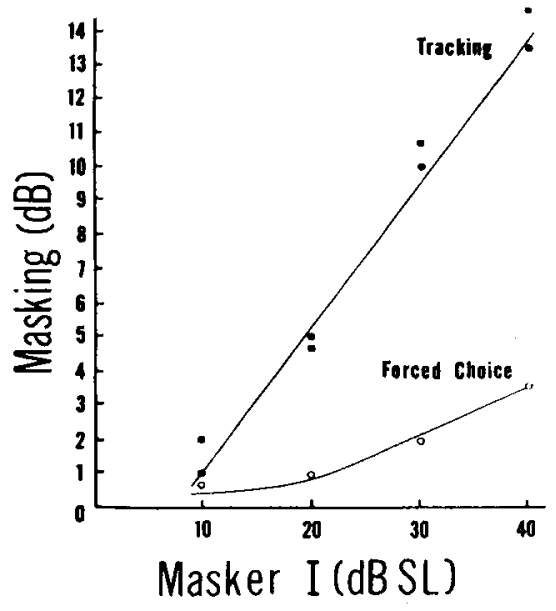

Fig. 1. Contralateral tactile masking as a function of masker intensity, measured by two methods.

160 judgments was obtained from each $S$ under each of the 20 conditions of the experiment over 16 sessions.

\section{Results and Discussion}

The proportion of correct judgments for each of the 20 conditions was converted to a $d^{\prime}$ value by referring to the tabled values provided by Swets (1964). To estimate the threshold in decibels for each of the five masking conditions, $d^{\prime}$ was plotted as a function of the four test-stimulus intensity values and a smooth curve was fitted by inspection to the four data points. The threshold was defined as the stimulus intensity that corresponded to a $\mathrm{d}^{\prime}$ value of .95 (75\% correct judgments). This procedure was employed for the data obtained under each of the five masking conditions. The amount of masking, specified as the difference in decibels between the threshold in the presence of the masker and the threshold in the absence of the masker, is plotted in Fig. 1 against the intensity level of the masking stimulus. In addition, the data obtained by Sherrick (1964) using the threshold-tracking procedure are plotted in Fig. 1 (filled points). The greater amount of masking obtained by Sherrick suggests that in the threshold-tracking task S's criterion, as well as stimulus detectability, was affected by the masker. Furthermore, the extreme divergence of the curves obtained under the two methods suggests that the magnitude of the criterion shift in the threshold-tracking task increased as the masker intensity increased. Sherrick's results seem to be highly reliable since we were able to obtain almost identical amounts of masking using the threshold-tracking method (filled squares).

\section{EXPERIMENT 2}

In Experiment 2 the tracking precedure used by Sherrick (1964) was modified in an attempt to eliminate the effects of criterion shifting on the measurement of masked thresholds. In the threshold-tracking experiment a stimulus is presented to $S$, and he is required to press a switch until the sensation disappears and then to release it until the sensation reappears. Each stimulus presentation can be thought of as a sample from the signal-plus-noise (SN) distribution. If a particular observation exceeds his criterion $S$ will decrease stimulus intensity, but if the observation is below his criterion $S$ will increase stimulus intensity. The higher $S$ sets his criterion, the higher the estimation of his threshold will be. In the present study this procedure was designated SN tracking. When using this method, Ss often complain that a contralateral masking vibration paired with a test stimulus is difficult to evaluate because they can't remember what the masker alone feels like. It is not surprising that they set very high criteria for detecting the stimulus.

A tracking procedure was devised in which the stimulus was presented during the first of two successive observation intervals (sample from the SN distribution) and no stimulus was presented during the second observation interval (sample from the $\mathbf{N}$ distribution), enabling $\mathbf{S}$ to compare directly the sensation of the masker with the sensation of the masker paired with the test stimulus. This arrangement is very similar to that described by Sherrick (1963). The S was instructed to decrease stimulus intensity if the observation in the first interval was greater than the one in the second interval, and to increase stimulus intensity if the observation in the first interval containing the stimulus was equal to or less than the observation in the second interval containing no stimulus. By this procedure, designated SN-N tracking, it was thought that the excessive criterion shifting induced by the masking stimulus might be eliminated.

The purpose of Experiment 2 was to compare the amount of masking obtained under the SN-N tracking method with that obtained under the SN tracking procedure when the masker is applied to an ipsilateral fingertip and when the masker is applied to a contralateral fingertip.

\section{Method}

Subjects. A student and a faculty member at Hamilton College served as Ss. Prior to the beginning of Experiment 2, several sessions were devoted to practice in $\mathrm{SN}$ and $\mathrm{SN}-\mathrm{N}$ tracking under ipsilateral and contralateral tactile masking conditions.
Apparatus. Stimuli were brief mechanical pulses produced by applying to the vibrator a 1-msec square-wave electrical pulse generated by a pulse generator (Tektronix 161). The response of the vibrator to a $1-\mathrm{msec}$ square-wave pulse is an initial large positive displacement of the vibrator contactor followed by a large negative displacement and a series of smaller positive and negative displacements (Sherrick, 1964). The duration of the major activity is approximately $10 \mathrm{msec}$, and a single pulse is felt as a brief tap.

A pulse from one pulse generator, after passing through an impedance-matching transformer and a graphic-recording attenuator, was applied to the vibrator in contact with the test area at the right index fingertip. The pulse from another pulse generator, after passing through a decade attenuator box and an impedance-matching transformer, was applied to a vibrator which provided the masking stimulus to a fingertip either ipsilateral or contralateral to the test area. For the SN tracking method the masker and the test stimulus were presented together every 2 sec over a tracking period of $1 \mathrm{~min}$. A $1-\mathrm{msec}$ flash produced by the horizontal sweep of an oscilloscope was used to indicate to $S$ the observation interval in which the stimulus was presented.

In the SN-N tracking condition, a second 1 -msec observation interval containing no test stimulus but only the masker followed the first interval containing both masker and test stimulus. In SN-N tracking the sequence of an observation interval containing masker and test stimulus followed $.6 \mathrm{sec}$ later by a second observation interval containing only the masker was repeated every $2 \mathrm{sec}$ over a tracking period of $1 \mathrm{~min}$.

Procedure. The $\mathrm{S}$ was instructed to track his threshold by operating a pedal attached to the recording attenuator. When the pedal was depressed, stimulus intensity decreased at a rate of $1 \mathrm{~dB} / \mathrm{sec}$, and when it was released, stimulus intensity increased at the same rate. The S's manipulation of intensity level was recorded as a function of time by the graphic recording attenuator. Masking was measured under four conditions of tracking: SN tracking with an ipsilateral masker, $S N$ tracking with a contralateral masker, $\mathrm{SN}-\mathrm{N}$ tracking with an ipsilateral masker, and $\mathrm{SN}-\mathrm{N}$ tracking with a contralateral masker. During a session $\mathrm{S}$ tracked his threshold under one of these four conditions. The order of the conditions was counterbalanced over successive sessions. Within a session thresholds were obtained when the masking stimulus was $10,20,30$, and $40 \mathrm{~dB}$ SL and when no masker was presented. The order in which the masker 


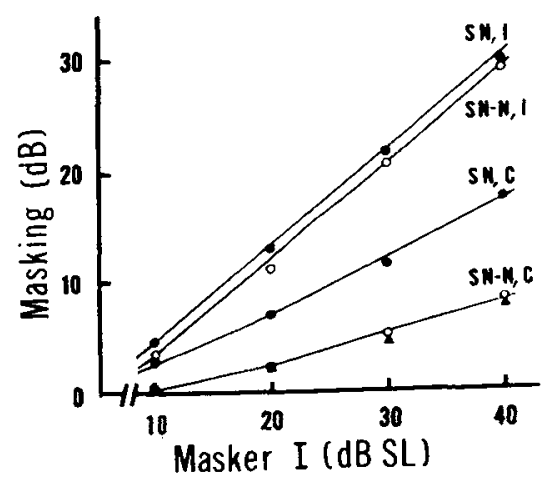

Fig. 2. Contralateral and ipsilateral tactile masking as a function of masker intensity, measured by $\mathrm{SN}$ and $\mathrm{SN}-\mathrm{N}$ threshold tracking methods.

intensities occurred within a session was random, and before and after the measurement of each masked threshold a threshold without the masker was measured. During the experiment six measurements of the amount of masking at each masker intensity under each of the four tracking conditions were obtained for each of the Ss.

In the contralateral tactile masking condition, the test stimulus was applied to the right index fingertip and the masking stimulus was applied to the ring fingertip of the left hand. When the masker was ipsilateral, the test stimulus was again applied to the index fingertip of the right hand but the masking stimulus was applied to the ring fingertip of the same hand.

\section{Results and Discussion}

In Fig. 2 the amount of masking is plotted as a function of masker sensation level for each of the four tracking conditions. The amount of contralateral tactile masking was considerably greater for $\mathrm{SN}$ tracking than for SN-N tracking. The fact that the amount of contralateral masking obtained by SN-N tracking (open circles) was essentially the same as that obtained in a subsequent experiment in which the forced-choice method was used (filled triangles) supports the hypothesis that SN-N tracking yields a measure of masking uncontaminated by the effects of criterion shifting. Ipsilateral tactile masking was considerably greater than contralateral tactile masking. The greater opportunity for neural interaction when both vibrators were on fingertips of the same hand rather than on fingertips of different hands seems to be the most plausible explanation of this finding. The amount of ipsilateral masking measured by SN tracking was only about $1 \mathrm{~dB}$ more than the amount measured by SN-N tracking. It appears that ipsilateral tactile masking is almost entirely a function of decreased stimulus detectability. The reduction in stimulus detectability produced by the masker may have been so great that criterion shifting by $S$ as a determinant of masking became relatively insignificant. On the other hand, when the reduction in stimulus detectability produced by the masker is small, as was the case with contralateral tactile masking, criterion shifting becomes a more significant determinant of masking if $S$ is unable to compare directly the sensation of the masker with that of the masker paired with the test stimulus.

Contralateral tactile masking, when measured by classical psychophysical techniques, appears to be determined by upward criterion shifting as well as by reduced stimulus detectability. No independent measures of S's criterion were obtained in the present study, but Gescheider et al (1969) found that auditory maskers tend to induce $S$ to raise his criterion for detecting tactile stimuli. This finding prompted us to propose the criterion shifting hypothesis as an explanation of our findings for contralateral tactile masking.

The fact that as much as $10 \mathrm{~dB}$ more contralateral tactile masking was obtained by $\mathrm{SN}$ tracking than by $\mathrm{SN}-\mathrm{N}$ tracking or the forced-choice method suggests that criterion shifting corresponds to S's working on basically different tasks when methods of threshold measurement are changed. In the SN-N tracking and forced-choice tasks, where $S$ can compare the masker alone with the masker plus the test stimulus, he experiences a change in the overall stimulus pattern before the test stimulus is intense enough to be perceived at the test site. Under these conditions the masked thresholds should be lower than those obtained by classical methods where such direct comparisons of masker and masker plus test stimulus are impossible. In SN tracking, for example, a sensation at the test site must be detected rather than a change in the overall pattern of stimulus, and masked thresholds are relatively high. The amount of contralateral tactile masking could be specified from performance on either of the two tasks, pattern recognition or stimulus detection, but the choice of task would depend upon the definition of masking employed in the particular investigation.

\section{EXPERIMENT 3}

The purpose of Experiment 3 was to determine whether $\mathrm{SN}$ and $\mathrm{SN}-\mathrm{N}$ tracking $y$ ield different amounts of auditory masking when a test tone and a noise background are presented to the same ear. Because under this condition a great deal of peripheral masking occurs, it was expected that criterion shifting would be a minor contributor to masking. Therefore, the amount of masking measured by SN tracking and SN-N tracking were not expected to greatly differ.

\section{Method}

Subjects. Four male Hamilton college students and one faculty member served as Ss. Prior to the experiment a few sessions were devoted to training the $S s$ in the use of the $\mathrm{SN}$ and $\mathrm{SN}-\mathrm{N}$ procedures for tracking auditory thresholds.

Apparatus. The 1,000-Hz output of an audio oscillator (General Radio 1304-B), after passing through an impedance-matching transformer, a graphic-recording attenuator (Grason-Stadler E3262A), and a second impedance-matching transformer, became the input to Channel 1 of an electronic switch (Grason-Stadler 829E). The white-noise output of a noise generator (Grason-Stadler 901B), after passing through an impedance-matching transformer and a decade attenuator box, became the input to Channel 2 of the electronic switch. The output of Channels 1 and 2 of the electronic switch were applied to an earphone (Permoflux PDR-600). Rise and decay time was $25 \mathrm{msec}$. The duration of the tone/noise burst, controlled by two pulse generators (Tektronix 161), was 250 msec. In SN tracking the tone/noise burst was presented every $2.5 \mathrm{sec}$ for $250 \mathrm{msec}$ over a $1-\mathrm{min}$ tracking period. The deflection of a voltmeter needle was used to indicate to $S$ the observation interval in which the tone/noise burst was presented.

In SN-N tracking a second 250-msec observation interval containing only the noise burst followed the first. The sequence of the $250 \mathrm{msec}$ tone/noise observation interval, followed $250 \mathrm{msec}$ later by the second $250-\mathrm{msec}$ observation interval containing only the noise, was repeated every $2.5 \mathrm{sec}$ over a period of 1 min.

Procedure. During an experimentàl session $\mathrm{S}$ tracked auditory thresholds under either the $\mathrm{SN}$ or the $\mathrm{SN}-\mathrm{N}$ tracking conditions, the order of presentation of which was counterbalanced over sessions. The amount of auditory masking of the $1,000-\mathrm{Hz}$ tone determined when the white noise measured by a sound-level meter (General Radio 1551-C) and an earphone coupler (General Radio 1560-P82) was 20 , $30,40,50,60,70,80$, and $90 \mathrm{~dB}$ SPL. Before and after each masked threshold measurement, the unmasked threshold was measured and masking was taken as the difference between the unmasked and masked thresholds. During the experiment two measurements of masking for each of the eight masker intensities under each of the two tracking methods were obtained. Results and Discussion

Figure 3 shows the effect of increasing 


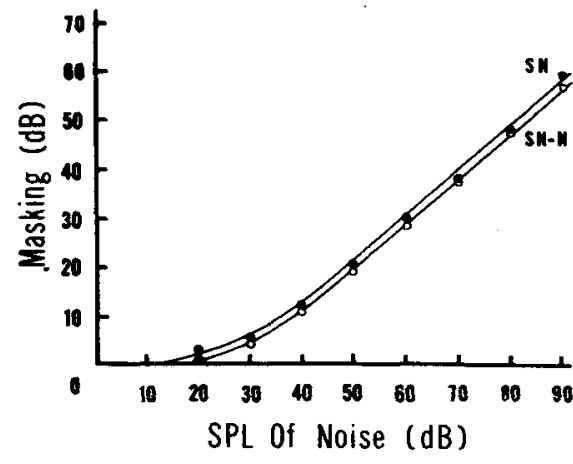

Fig. 3. Auditory masking as a function of masking noise intensity, measured by SN and SN-N threshold tracking methods.

the intensity of white noise on the threshold for a $1,000-\mathrm{Hz}$ tone. Beyond $30 \mathrm{~dB}$ the amount of masking was a linear function of the intensity of the noise. The slope of the function is approximately 1.0 , indicating that each additional decibel of noise produces an additional decibel of masking. The function obtained by SN tracking corresponds almost exactly to one plotted by Hirsh (1952) from data of Hawkins and Stevens (1950), who determined thresholds by the method of adjustment. Although forced-choice data were not obtained in the present experiment, the results suggest that there would be only slightly less masking obtained by a forced-choice method than by one of the classical psychophysical methods.

The primary finding of Experiment 3 was that the amount of auditory masking obtained by SN tracking is little more than a decibel greater than that obtained by SN-N tracking. Thus, the effects of threshold tracking method are negligible for both ipsilateral tactile masking and ipsilateral auditory masking. Consideration of the results from the perspective of the conceptual framework elaborated in the previous experiments suggests that both of these kinds of masking result in rather large reductions in stimulus detectability and only small criterion shifts. Ipsilateral auditory masking may be almost entirely attributable to reduced stimulus detectability but contralateral auditory masking, if it is at all analogous to contralateral tactile masking, might be reduced greatly by employing, SN-N tracking or forced-choice methods.

\section{EXPERIMENT 4}

Intermodality masking of touch by hearing has been found to be a combination of reduced stimulus detectability and upward criterion shifting (Gescheider et al, 1969). The purpose of Experiment 4, therefore, was to compare the amount of auditory-tactile masking obtained under the SN and SN-N tracking methods. Since criterion shifting is an important part of auditory-tactile masking, considerably more masking should be obtained by the SN method than by the SN-N method.

\section{Method}

Subjects. Two students and one faculty member at Hamilton College, all with extensive experience in tactile threshold tracking, served as Ss.

Apparatus. Square-wave electrical pulses having a duration of $1 \mathrm{msec}$ were applied to the vibrator and to a pair of earphones (Permoflux PDR-600) to produce the tactile test stimulus and the auditory masking stimulus, respectively. The same apparatus used in Experiment 2 was used in Experiment 4 except that the masker signals were applied to the earphones rather than to a vibrator.

Procedure. During the experiment four measurements of masking were obtained from each $\mathrm{S}$ when the auditory masker was $30,45,60,75$, and $90 \mathrm{~dB}$ SL under both SN tracking and SN-N tracking methods. As in the previous experiments, the tracking method was counterbalanced over sessions, masker intensity was randomized within sessions, and an unmasked threshold was measured before and after the measurement of each masked threshold.

\section{Results and Discussion}

Figure 4 shows that auditory-tactile masking increases as an approximately linear function of auditory masker intensity when both are specified in decibels. The amount of masking obtained by $\mathrm{SN}$ tracking corresponds almost exactly to the amount obtained under comparable stimulus conditions by Gescheider and Niblette (1967, open squares) using the same method of threshold measurement. In the present experiment, however, considerably less masking was obtained when the SN-N tracking method was employed. The amount of auditory-tactile masking measured by the forced-choice method and reported in an earlier paper (Gescheider et al, 1969) is plotted in Fig. 4 as open circles. The close correspondence between the amount of masking obtained by the $\mathrm{SN}-\mathrm{N}$ tracking and forced-choice methods constitutes strong support for the hypothesis that auditory-tactile masking measured by SN-N tracking is not influenced by criterion shifting.

\section{REFERENCES}

CRAIG, J. C. Vibrotactile spatial summation Perception \& Psychophysics, 1968, 4, 351-354.

GESCHEIDER, G. A., BARTON, W. G., BRUCE, M. R., GOLDBERG, J. M., \& GREENSPAN,

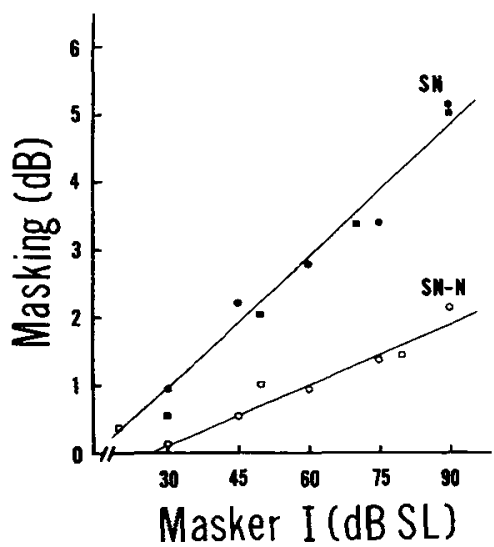

Fig. 4. Auditory-tactile masking as a function of the intensity of the auditory masker, measured by $\mathrm{SN}$ and $\mathrm{SN}-\mathrm{N}$ threshold-tracking methods.

M. J. The effects of simultaneous auditory stimulation upon the detection of tactile stimuli. Journal of Experimental Psychology, $1969,81,120-125$.

GESCHEIDER, G. A., \& NIBLETTE, R. K. Cross-modality masking for touch and hearing. Journal of Experimental Psychology, 1967. 74, 313-320.

GESCHEIDER, G. A., \& WRIGHT, J. H. Effects of sensory adaptation on the form of the psychophysical magnitude function for cutaneous vibration. Joumal of Experimental Psychology, 1968, 77, 308-313.

GILSON, R. D. Vibrotactile masking: Some spatial and temporal aspects. Perception \& Psychophysics, 1969, 5, 176-180.

HAWKINS, J. E., JR., \& STEVENS, S. S. The masking of pure tones and of speech by white noise. Journal of the Acoustical Society of America, 1950, 22, 6-13.

HIRSH, 1. J. The measurement of hearing. New York: McGraw-Hill, 1952.

SHERRICK, C. E., JR. Temporal relations in the interaction of pressure pulses. Princeton Cutaneous Project, Progress Report No. 2, 1963.

SHERRICK, C. E., JR. Effects of double simultaneous stimulation of the skin. American Journal of Psychology, 1964, 77, 42-53.

SWETS, J. A. (Ed.) Signal detection and recognition by human observers New York: Wiley, 1964. Pp. 659-683.

UTTAL, W. R., \& COOK, L. Systematics of the evoked somatosensory cortical potential: A p sych ophysical-electrophy siological comparison. Annals of the New York Academy of Sciences, 1964, 112, 60-80.

UTTAL, W. R., \& Smith, P. Contralateral and heteromodal interaction effects in somatosensation: Do they exist? Perception \& Psychophysics, 1967, 2, 363-368.

\section{NOTES}

1. This research was supported by United States Public Health Service Grant NB-07620 from the National Institute of Neurological Diseases and Stroke.

2. Address: Department of Psychology, Hamilton College, Clinton, New York 13323.

(Accepted for publication April 12, 1970.) 